\title{
Effects of SARS-CoV-2 mRNA vaccines on platelet polyphosphate levels and inflammation: A pilot study
}

\author{
TAKASHI UEMATSU ${ }^{1}$, ATSUSHI SATO ${ }^{1}$, HACHIDAI AIZAWA ${ }^{1}$, TETSUHIRO TSUJINO ${ }^{1}$, \\ TAISUKE WATANABE ${ }^{1}$, KAZUSHIGE ISOBE ${ }^{1}$, HIDEO KAWABATA ${ }^{1}$, YUTAKA KITAMURA ${ }^{1}$, \\ TAKAAKI TANAKA ${ }^{2}$ and TOMOYUKI KAWASE ${ }^{3}$ \\ ${ }^{1}$ Tokyo Plastic Dental Society, Tokyo 114-0002; ${ }^{2}$ Department of Materials, Science and Technology, \\ Niigata University, Niigata 950-2181; ${ }^{3}$ Division of Oral Bioengineering, \\ Niigata University Graduate School of Medical and Dental Sciences, \\ Niigata 951-8514, Japan
}

Received November 29, 2021; Accepted January 10, 2022

DOI: 10.3892/br.2022.1504

\begin{abstract}
Platelets function as immune cells in conjunction with white blood cells, targeting invading pathogens and inducing immune reactions. Intercellular communications among these immune cells are partly mediated by platelet polyphosphate (polyP), which was originally recognized as a thrombotic and hemostatic biomolecule. To determine the involvement of polyP in SARS-CoV-2-mRNA vaccine-induced immune responses, specifically in inflammatory responses, the effects of mRNA vaccines on platelet polyP levels were examined. Before and after vaccination with the COVID-19 vaccine (BNT162b2), blood samples were obtained from healthy, non-smoking individuals who did not have any systemic diseases. Test group demographics skewed somewhat towards either older males (first vaccination, $\mathrm{n}=6$; second vaccination, $n=8$ ) or younger females (first vaccination, $n=14$; second vaccination, $n=23$ ). polyP levels in washed platelets from the blood samples were determined using the fluorometric method with DAPI. The side-effects of vaccination were recorded as scores. In the female group, platelet polyP levels decreased after the first vaccination, and the side-effect score increased after the second vaccination. Moderate correlation coefficients were observed between the reduction in polyP levels and the side-effect scores and pre-vaccination polyP levels. Despite the small sample size, this pilot study suggests that platelet polyP may suppress the side effects induced by the mRNA vaccines after the first vaccination, but not the second vaccination in younger female subjects, who generally have higher immune responsiveness than their male counterparts.
\end{abstract}

Correspondence to: Dr Tomoyuki Kawase, Division of Oral Bioengineering, Niigata University Graduate School of Medical and Dental Sciences, 2-5274 Gakkocho-dori, Chuo-Ku, Niigata 951-8514, Japan

E-mail: kawase@dent.niigata-u.ac.jp

Key words: COVID-19, mRNA vaccine, blood platelet, polyphosphates, sex, side effects

\section{Introduction}

Platelets are involved in multiple immune responses, including hemostasis, thrombosis and wound healing. However, recent studies have highlighted their distinct functions in inflammatory responses. Upon infection, platelets first bind to infectious pathogens, such as bacteria or viruses. Then, the platelets secrete various immunoregulatory cytokines and express receptors to mediate various immune effects and regulatory functions $(1,2)$, including regulation of macrophage functions, stimulation or inhibition of regulatory $\mathrm{T}$ cells, and secretion of pro-resolving mediators $(3,4)$. However, their regulatory functions can be complex: Both pro-inflammatory and anti-inflammatory regulations are modulated by the degree and phase of injury and inflammation, which vary on a case-by-case basis (2-6).

The novel severe acute respiratory syndrome coronavirus 2 (SARS-CoV-2) is the causative agent of the current coronavirus disease-2019 (COVID-19) pandemic. The virus continues to pose a significant threat to global health. Like most other coronaviruses, SARS-CoV-2 is comprised of the standard biological components, including an envelope, a membrane and spike proteins (7). The spike protein recognizes and binds to the host cell surface receptor, angiotensin-converting enzyme 2 (ACE2), which facilitates entry of SARS-CoV-2 into host cells (7), including platelets. It is hypothesized that the behavior of the virus is similar to the influenza virus $(1,8,9)$. Initially, coronavirus infection elevates fibrinogen and D-dimer levels to evoke systemic hypercoagulability and frequent venous thromboembolic events (10). This could be considered a severe proinflammatory state, which consequently induces pro-coagulopathy through endothelial activation or damage (10). As anticipated, recent studies have found that the number of activated, circulating platelets increase in COVID-19 patients (10-13). This suggests that platelets may contribute to COVID-19 severity (13), although the role of platelets in the pathogenesis of SARS-CoV-2 is not yet well understood.

Several COVID-19 vaccines are now widely available and others are still in production. The World Health Organization 
(WHO) and the U.S. Food and Drug Administration (FDA) approved the Pfizer/BioNTech mRNA vaccine (BNT162b2) as one of the first COVID-19 vaccines on the 23rd of August, $2021(14,15)$. Due to these approvals, COVID-19 vaccines based on mRNA technology dominate the seven major markets (7MM), comprised of the US, Germany, France, Italy, Spain, the UK and Japan, which held a collective market share of $95.4 \%$ in 2021 (16). Although the market shares vary among individual countries at present (17), like other major countries including other members of the 7MM, the Japanese government approved and provided the Pfizer/BioNTech vaccine, first to medical staff as a priority followed by older citizens. The Moderna mRNA vaccine (mRNA-1273) has since been approved as the second major vaccine, now widely available to all applicants.

Both the Pfizer/BioNTech and Moderna mRNA vaccines are designed to produce the spike protein of SARS-CoV-2 and then to induce formation of spike protein-specific neutralizing antibodies, which are expected to protect against infection by the target viruses through immunological reactions $(18,19)$. As these vaccines mimic the initial phase of reaction to SARS-CoV-2 infection, these agents can also cause various side-effects that are consistent with the symptoms of infection by SARS-CoV-2 (20). Thus, it is possible that the vaccines stimulate platelet activation and degranulation, to modify inflammation and immunological reactions, likely involving polyphosphate (polyP).

Among platelet regulatory molecules, platelet polyP has recently received increasing attention, not only as a coagulation factor, but also as a potent modulator of inflammation (21-23). polyP is a linear polymer of orthophosphates linked by high-energy phospho-anhydride bonds, and is stored primarily in platelet dense granules and released upon activation of platelets $(21,24)$. polyP can act as a proinflammatory factor or an anti-inflammatory factor by triggering bradykinin release and inhibiting complement activation, respectively $(25,26)$. Thus, it is hypothesized that platelets, activated by infection by pathogens, release pro-inflammatory and anti-inflammatory biomolecules, including polyP, thereby exacerbating or suppressing inflammation, respectively. To determine the possible involvement of platelet polyP in the COVID-19 mRNA vaccine-induced side-effects, including the immune responses, the effects of the COVID-19 mRNA vaccine on platelet polyP levels were examined.

\section{Materials and methods}

Preparation of the platelet suspensions. The study design and consent forms for all procedures (approval no. 2019-0423) were approved by the Ethics Committee for Human Participants at the Niigata University School of Medicine (Niigata, Japan), and complied with the Helsinki Declaration of 1964, as revised in $2013(27,28)$.

Vaccination with mRNA vaccines, BNT162b2 and mRNA-1273, was controlled by the Japanese government and local governments (29). In principle, the residents were categorized into several groups depending on various factors, such as age, severity of pre-existing diseases, area of residence and type of occupation. The government scheduled vaccinations without or upon the requests of individuals, and designated a vaccination date. The first priority for vaccination was given to medical workers, ambulance crews and public health center officials.

Healthy Japanese subjects (male: first vaccination, $\mathrm{n}=6$, median age, 55.5, age range, 47-60); second vaccination, $\mathrm{n}=8$, median age, 55.0, age range, 47-72; and female: first vaccination, $\mathrm{n}=14$, median age, 29.5, age range, 20-57; second vaccination, $\mathrm{n}=23$, median age, 32.0 , age range, 21-66), who were selected from medical workers that were not receiving continuous medical treatment and would be receiving the Pfizer/BioNTech vaccine (BNT162b2) participated in this study. As for the inclusion criteria, the subjects were required to have no limitations on their activities of daily living, regardless of lifestyle-related diseases or regular medication. Subjects who had cancer or received estrogen replacement therapy were excluded. All subjects were required to declare that they were free of human immunodeficiency virus, hepatitis B virus, hepatitis $\mathrm{C}$ virus, or syphilis infections; any subjects who had been infected with any of these viruses were excluded from the study. No acute reactions were observed in the subjects during or immediately after blood samples were taken.

The age distributions of the sample groups and the participants' constitutions (male numbers vs. female numbers) are shown in Fig. 1. The female groups (first dose, $36.3 \pm 5.0$ years; second dose, $36.3 \pm 12.3$ years, mean $\pm \mathrm{SD}$ ) were significantly younger than the respective male groups (first dose, $56.8 \pm 8.8$ years; second dose, $56.0 \pm 7.8$ years). All participants (male, $\mathrm{n}=6$; female, $\mathrm{n}=14$ ) provided blood samples after both the first and second vaccinations. Additional participants (male, $n=2$; female, $n=9$ ) provided blood samples only after the second vaccination.

When the participants were available during the daytime on weekdays, after an appropriate interval of time following receipt of the vaccine (first vaccination, 4-10 days, median, 8 days; second vaccination, 4-15 days, median, 7 days), blood withdrawal was performed by venipuncture of the median cubital vein using $21 \mathrm{G}$ wing needles (NIPRO). Blood was collected in vacutainer A-formulation of acid-citrate-dextrose (ACD-A) glass tubes (BD Biosciences). Blood cells from each sample were counted using an automated hematology analyzer (pocH-100i V Diff; Sysmex) and intermittently rotated at $22-25^{\circ} \mathrm{C}$ until use.

Prior to centrifugation, $0.5 \mathrm{ml}$ ACD-A (Terumo) was added to each blood sample, then the samples were incubated for $10 \mathrm{~min}$ to prevent unexpected coagulation, which is often observed in female blood samples (30). Platelets were collected using the dual-spin protocol of pure-platelet rich plasma (PRP) preparation (1st soft spin: $472 \mathrm{x}$ g for $10 \mathrm{~min}$; 2nd hard spin: $578 \mathrm{x}$ g for $5 \mathrm{~min}$, at $22-25^{\circ} \mathrm{C}$ ). The resulting platelet pellets were then gently suspended in PBS, incubated for $15 \mathrm{~min}$ at $22-25^{\circ} \mathrm{C}$, and fixed with ThromboFix Platelet Stabilizer (Beckman-Coulter, Inc.). The fixed samples were stored at $4^{\circ} \mathrm{C}$ for at least $24 \mathrm{~h}$ prior to polyP quantification. It should be noted that the centrifugation conditions were mild, thus minimizing platelet activation among the preparation conditions performed (31).

Additionally, healthy Japanese subjects who received the Moderna vaccine (mRNA-1273) were selected (5 females; median age 37.0; age range 34-48 years), using the same inclusion and exclusion criteria as above. 
A

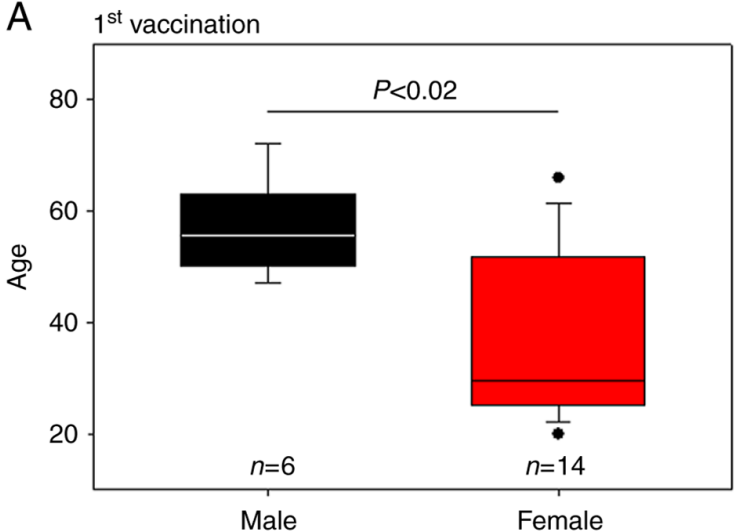

C

Number of samples
B

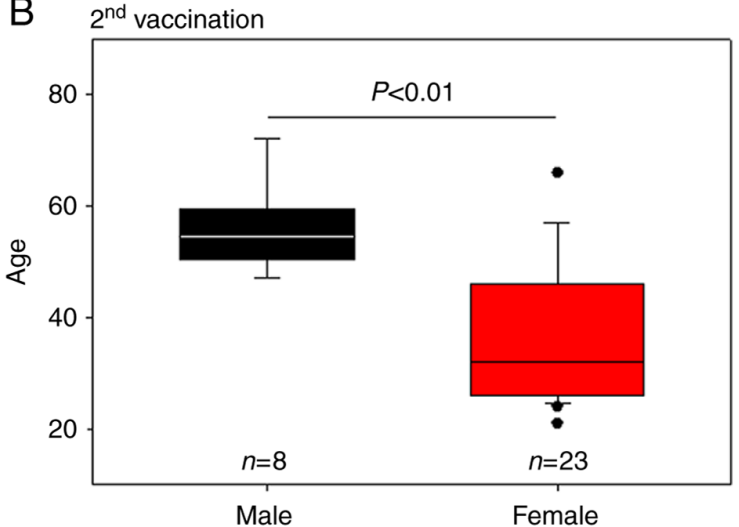

$1^{\text {st }}$ and $2^{\text {nd }}$ vaccination

Male: $n=6$, Female: $n=14$

Only $2^{\text {nd }}$ vaccination

Figure 1. Age distribution in the male and female groups. Distribution in age by sex (A) post-first and (B) post-second vaccination. The black and red columns represent males and females, respectively. The block dots represent outliers. (C) The sample population numbers.

Fluorometric measurement of platelet polyphosphate using $D A P I$. After storage at $4^{\circ} \mathrm{C}$ for $24 \mathrm{~h}$, the fixed platelets were centrifuged at $578 \mathrm{x} \mathrm{g}$ for $5 \mathrm{~min}$ at $22-25^{\circ} \mathrm{C}$, after which the supernatants were carefully aspirated. The resulting platelet pellets were gently suspended in Milli-Q water, as previous observations found that Milli-Q water does not increase background of fluorescence signal quantification, and enables DAPI to invade the cytoplasm without perforation (30). Platelets were counted using an automated hematology analyzer and then adjusted to the appropriate range $\left(<4.5 \times 10^{7} / \mathrm{ml}\right)$ for polyP quantification. DAPI (Dojindo Molecular Technologies, Inc.) was added to each tube at a concentration of $4 \mu \mathrm{g} / \mathrm{ml}$ and incubated for $30 \mathrm{~min}$ at $22-25^{\circ} \mathrm{C}$. Fluorescence intensity was measured using a fluorometer (FC-1; Tokai Optical Co., Ltd.) with excitation and emission wavelengths of 425 and $525 \mathrm{~nm}$, respectively (30).

Due to the lack of specific probes, it is difficult to accurately quantify platelet polyP levels in an efficient manner. Therefore, our methods are modeled using previous studies $(30,32,33)$ showing that the right shift of excitation and emission wavelengths (350 and $470 \mathrm{~nm}$ ) from those wavelengths used for DNA examination increases the specificity of DAPI against polyP, with some modifications. Specifically, quantification and visualization of platelet polyP using DAPI was optimized through the determination of the optimum light wavelengths for fluorescence, as well as for sample fixation and choice of the aqueous solution. Although the possible count of DAPI-bound, non-polyP substances may be included in the quantification process, this method enables rapid quantification of polyP levels without sacrificing recovery or reproducibility.

The polyP ratio was calculated for each individual, and all polyP ratios were combined for statistical analysis.

Determination of mitochondrial activity using a Cell Counting Kit-8 (CCK-8) assay. The number of platelets suspended in
PBS was adjusted to the density range of $2.5-5.0 \times 10^{7} / 100 \mu 1$, mixed with $10 \mu \mathrm{l}$ CCK-8 solution (Dojindo Molecular Technologies, Inc.), and incubated for $1 \mathrm{~h}$ at $22-25^{\circ} \mathrm{C}$. After centrifugation $\left(578 \mathrm{x} \mathrm{g}, 5 \mathrm{~min}, 22-25^{\circ} \mathrm{C}\right)$, the supernatants were analyzed using a spectrophotometer at $450 \mathrm{~nm}$ (SmartSpec Plus; Bio-Rad Laboratories, Inc.).

Evaluation of side-effects based on individual self-declarations. The score sheet of the side-effects are shown in Table I. The perception of vaccine side-effects was examined by asking the subjects the following questions: 'Have you had any of these side effects? Please score each side-effect on a scale where: 0 , not experienced; 1 , experienced; and 2, experienced strongly (more important or severe for you). If you took painkillers, please score 1. Also, please mention the number of days the side-effects lasted and the frequency of taking medicines treating them.' These questions were introduced as described in the questionnaire to the donors.

Statistical analysis. Quantification was performed in triplicate. Data are presented as the mean \pm standard deviation. Data plotted in box plots represent the median values, the upper and lower quartiles (box), and maximum and minimum values (whiskers). A Mann-Whitney U test was performed to compare the two groups (SigmaPlot version 13.0; Systat Software, Inc.). To confirm the statistical difference provided by the Mann-Whitney $U$ test, particularly given the fact that the score values were not continuous variables, data for the apparent reduction of platelet polyP levels $(<0.9)$ and the apparent side-effect scores (>8.2) in individual data were also analyzed using a Fisher's exact test. These border values were derived from the mean values of the female group's polyP ratio post-first vaccination $(0.9 \%)$ and the female group's score post-second vaccination $(8.2)$. $\mathrm{P}<0.05$ was considered to indicate a statistically significant difference. 
Table I. List of vaccine-induced side-effects and scoring of severity based on patient declarations.

Number of days or

Side-effects Severity score (a) doses of medicine taken (b) Final score ( $\mathrm{a}$ x b)

Systemic side-effects

Fever, temperature $>37.5^{\circ} \mathrm{C}$

Slight fever, temperature $37.2-37.5^{\circ} \mathrm{C} \quad 1$

Fatigue

Headache

Others including chills, arthralgia, myalgia, nausea, etc.

Local side-effects

Pain

Redness

Swelling

Others, including tenderness, itch, warmth, bruising, etc.

Allergic reaction

Rash

Skin burning

Red welts on face and lips

Analgesics dose

Type of analgesics ${ }^{\mathrm{a}}$
2

1

1

1

1

2
1
1

1

1

1

1

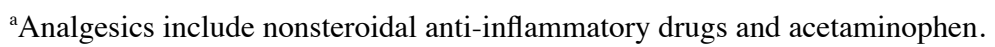

Table II. Platelet counts in whole-blood samples.

\begin{tabular}{lccrrr}
\hline & \multicolumn{2}{c}{ Male, $x 10^{4} / \mu 1$} & & \multicolumn{2}{c}{ Female, $\mathrm{x} 10^{4} / \mu 1$} \\
\cline { 2 - 3 } \cline { 5 - 6 } Vaccination status & First vaccination, $\mathrm{n}=6$ & Second vaccination, $\mathrm{n}=8$ & First vaccination, $\mathrm{n}=14$ & Second vaccination, $\mathrm{n}=23$ \\
\hline Pre-vaccination & $25.8 \pm 4.3$ & $26.0 \pm 4.4$ & - & $25.9 \pm 6.2$ & $26.9 \pm 8.3$ \\
Post-1st vaccination & $26.3 \pm 4.7$ & - & $25.7 \pm 4.6$ & $25.5 \pm 6.3$ & - \\
Post-2nd vaccination & - & & - & $24.8 \pm 6.0$ \\
\hline
\end{tabular}

Linear regression analysis and calculation of correlation coefficient values were performed. Absolute $\mathrm{R}$ values ranging from 0.6-0.79 and 0.4-0.59 were considered a 'strong' and 'moderate' correlation, respectively. $\mathrm{R}$ values of $0.2-0.39$ and $<0.2$ indicated a 'weak' and 'very weak' correlation, respectively.

\section{Results}

Initially, platelet counts were examined to detect thrombocytopenia before and after vaccination. As shown in Table II, no significant reduction following vaccination was found in any of the participant groups.

Ratios of polyP levels after vaccination (BNT162b2) to polyP levels pre-vaccination, that is, vaccine-induced reduction of polyP levels, are shown in Fig. 2A. There were no significant differences between the male and female groups after either the first vaccination $(1.104 \pm 0.290$ vs. $0.904 \pm 0.331)$ or the second vaccination $(1.028 \pm 0.204$ vs. $0.892 \pm 0.206)$. Scores for the side-effects in the post-vaccination period are shown in Fig. 2B. The scores were generally lower in the male groups than in the female groups; however, a significant statistical difference in symptoms between males and females $(\mathrm{P}<0.02)$ was found only after the second vaccination $(2.88 \pm 2.42$ vs. $8.22 \pm 6.20)$ (c.f., first vaccination, $1.67 \pm 1.21$ vs. $3.57 \pm 2.53)$ of the vaccine.

To confirm the statistical difference, the data were analyzed again using a Fisher's exact test. A statistically significant difference $(\mathrm{P}=0.042)$ between males and females was found in the polyP ratios after the first vaccination (Table III). Border values were adopted by the mean values of the post-first (polyP $=0.9 \%)$ and post-second vaccination $($ score $=0.82)$ scores in the female groups.

Scatter plots of the relationship between polyP ratios (pre-vaccination, as well as after the first and second doses of the vaccinations) and side-effect scores for BNT162b2 are shown in Fig. 3. In the male groups, the coefficient correlations (R) were 0.279 and -0.115 for the first and second vaccinations, respectively. Although the correlation value was very weak $(\mathrm{R}=0.142)$ for the second vaccination, a moderate correlation $(\mathrm{R}=0.556)$ was found for the first vaccination. 
Table III. Number of subjects showing apparent reduction of platelet polyphosphate and apparent exacerbation of vaccine side-effect scores following vaccination with the Pfizer-BioNTech vaccine.

\begin{tabular}{|c|c|c|c|c|c|}
\hline Vaccine dose & $\mathrm{n}$ & Platelet polyphosphate reduction $<0.9(\%)$ & P-value & Side-effect score $>8.2$ & P-value \\
\hline \multicolumn{6}{|l|}{$1 \mathrm{st}$} \\
\hline Male & 6 & $0(0.0)$ & $0.042^{\mathrm{b}}$ & $0(0.0)$ & - \\
\hline Female & 14 & $8(57.1)$ & & $0(0.0)$ & \\
\hline \multicolumn{6}{|l|}{ 2nd } \\
\hline Male & 8 & $3(37.5)$ & 0.412 & $0(0.0)$ & $0.032^{\mathrm{b}}$ \\
\hline Female & 23 & $14(60.9)$ & & $10(39.1)$ & \\
\hline
\end{tabular}

${ }^{\mathrm{a}}$ Fischer's exact test. ${ }^{\mathrm{b}} \mathrm{P}<0.05$.

A

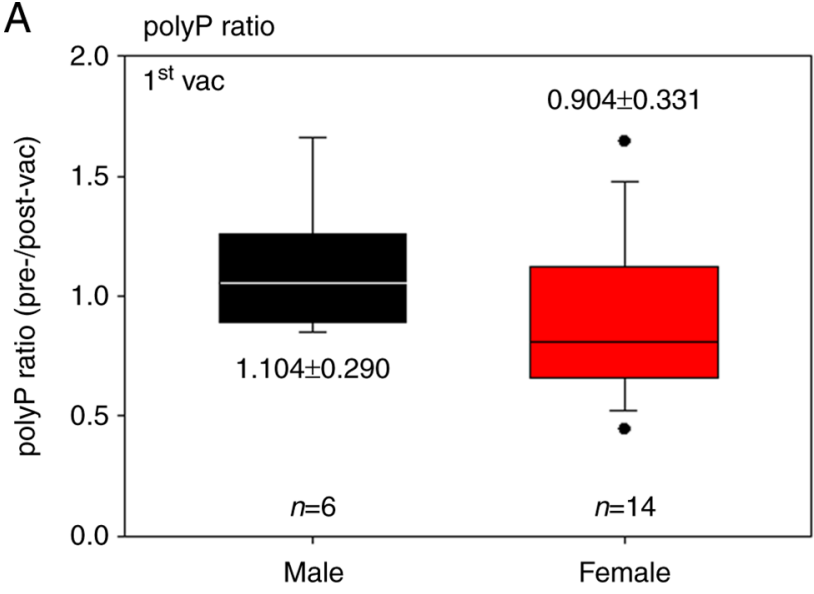

B

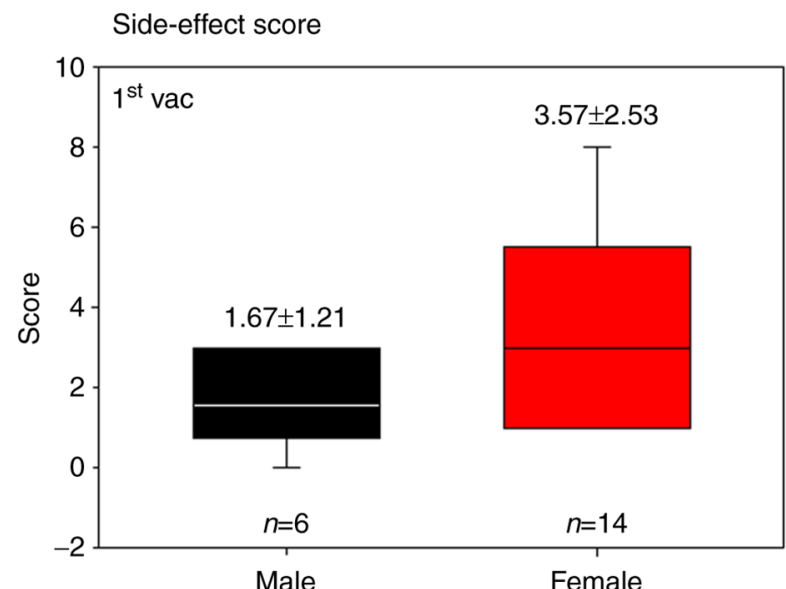

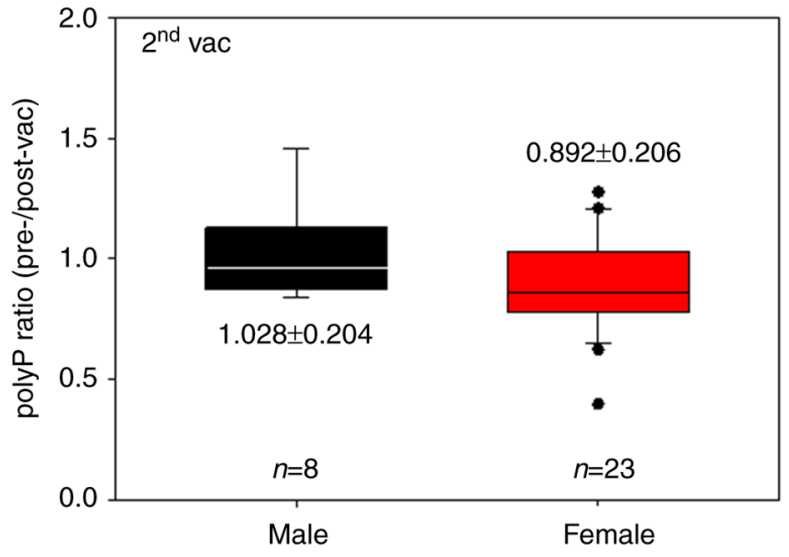

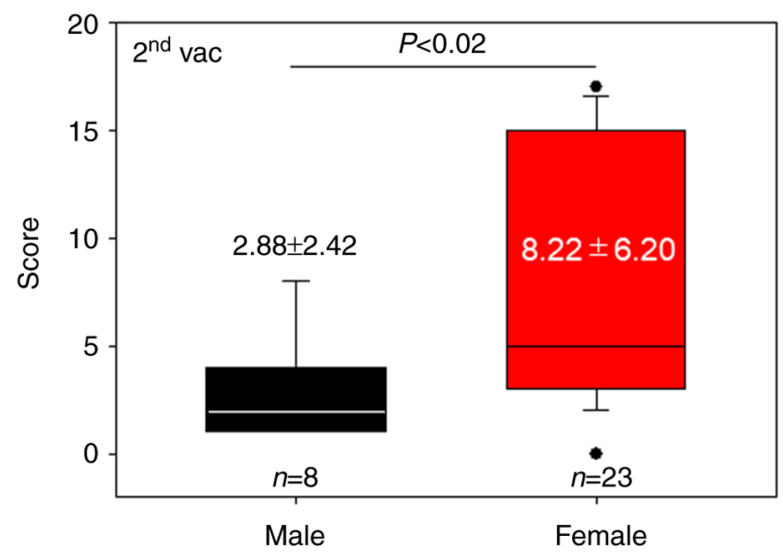

Figure 2. polyP ratio and side-effect score distributions in the male and female groups. (A) polyP ratio and (B) side-effect score distributions post-first and post-second vaccination in the males and females. The black dots represent outliers. Data are presented as the mean \pm SD. polyp, platelet polyphosphate.

Scatter plots of the relationship between polyP ratios and age in terms of the Pfizer/BioNTech vaccine (BNT162b2) are shown in Fig. 4. In the male group, the correlation coefficients were weak $(R=0.217)$ and very weak $(R=0.143)$ for the first and second vaccinations, respectively. In the female groups, the correlations were again negative but very weak $(R=-0.168)$ and weak $(\mathrm{R}=-0.224)$ for the first and second vaccinations, respectively.

Scatter plots of the relationship between the WST- 8 reduction and polyP levels, polyP ratios and pre-vaccination polyP levels for the Pfizer/BioNTech vaccine (BNT162b2) are shown in Fig. 5. Although polyP synthesis is not yet fully understood, several studies have suggested that mitochondria are involved in polyP synthesis in mammalian cells (34-36). To test this hypothesis, the mitochondrial activity and polyP levels were compared. In the group containing both male and female subjects, a strong correlation $(\mathrm{R}=0.651)$ was found between $\mathrm{NADH}$ activity, which is a proxy for mitochondrial activity, and platelet polyP levels (Fig. 5A). In the female group, after the first vaccination, a very strong negative correlation 

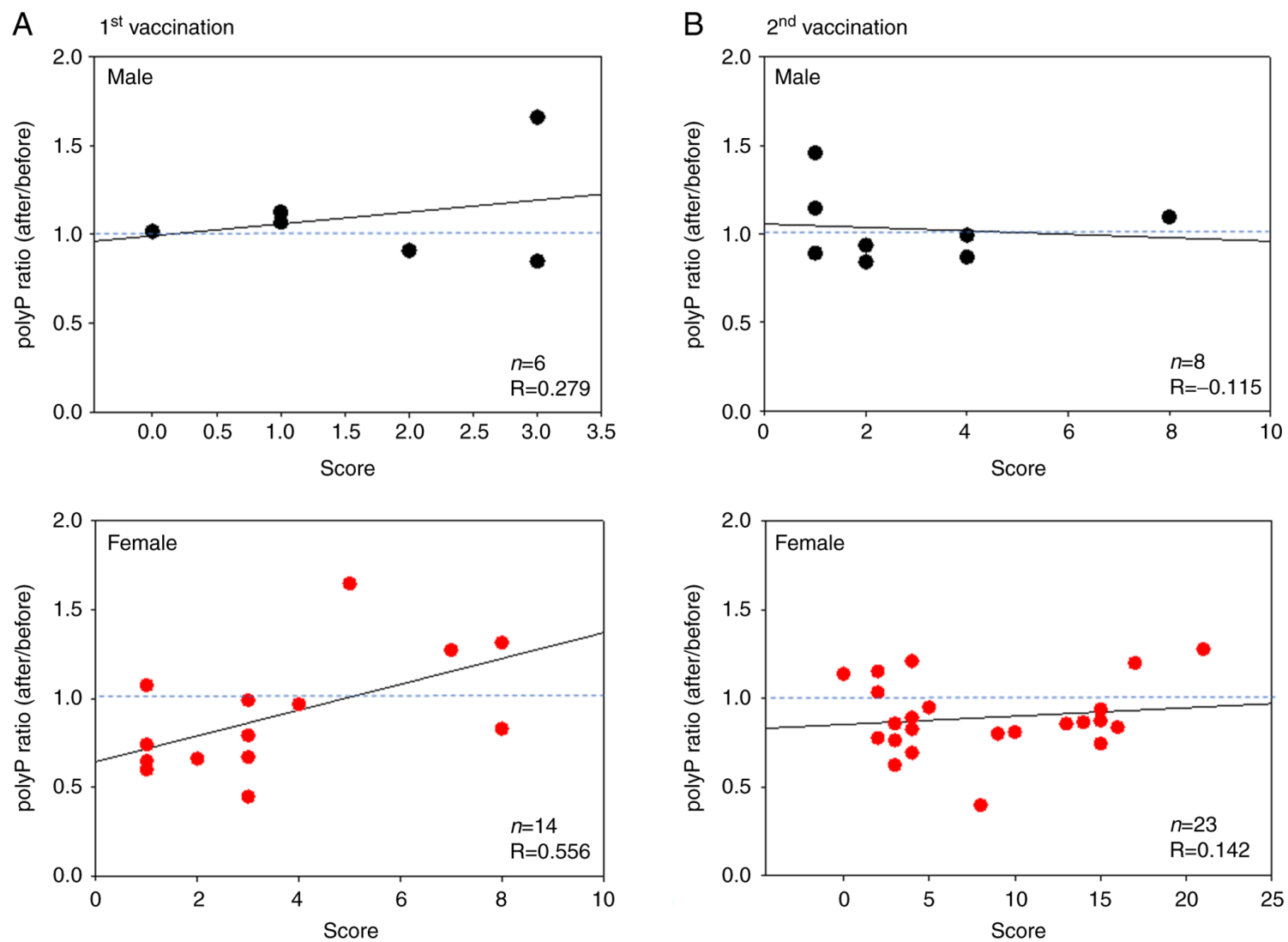

Figure 3. Scatter plots between polyP ratio and side-effect scores in the males and females. PolyP ratios of (A) post-first/pre-first vaccination and (B) post-second/pre-first vaccination are presented in individuals vaccinated with BNT162b2. polyp, platelet polyphosphate.
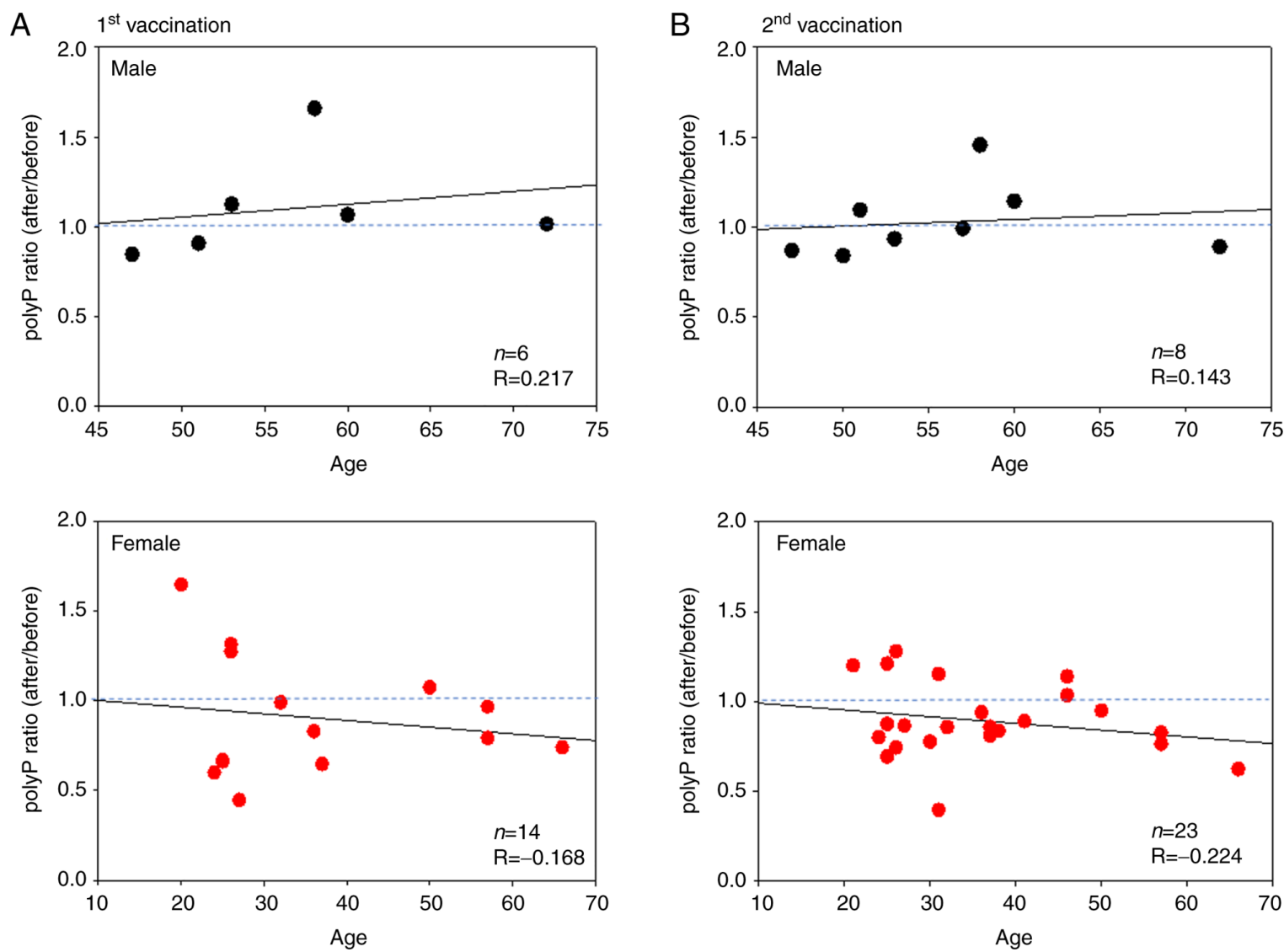

Figure 4. Scatter plot of the polyP ratios and age in the males and females. polyP ratios of (A) post-first/pre-first vaccination and (B) post-second/pre-first vaccination are presented in individuals vaccinated with BNT162b2. polyp, platelet polyphosphate. 
Table IV. polyP ratios and side effect scores in the patients treated with the Moderna vaccine.

\begin{tabular}{lclcccc}
\hline Subject & Age range & Sex & Initial dose polyP ratio & Side-effect score & Second dose polyP ratio & Side-effect score \\
\hline A & $55-59$ & Male & 1.32 & 1 & 1.423 & 2 \\
B & $30-34$ & Female & 0.817 & 6 & 1.286 & 24 \\
C & $55-59$ & Female & 0.785 & 3 & 0.742 & $8^{\text {a }}$ \\
D & $45-49$ & Female & 0.704 & $4^{\mathrm{a}}$ & 1.205 & 8 \\
E & $35-39$ & Female & 1.131 & 11 & Declined $^{\mathrm{b}}$
\end{tabular}

${ }^{a}$ Apparent delayed cutaneous reactions at and around the injection site (37), 'COVID arm', was observed. ${ }^{\mathrm{b}}$ Because of a high-risk, close contact with a COVID-19 patient, this subject was isolated and follow-up was discontinued.
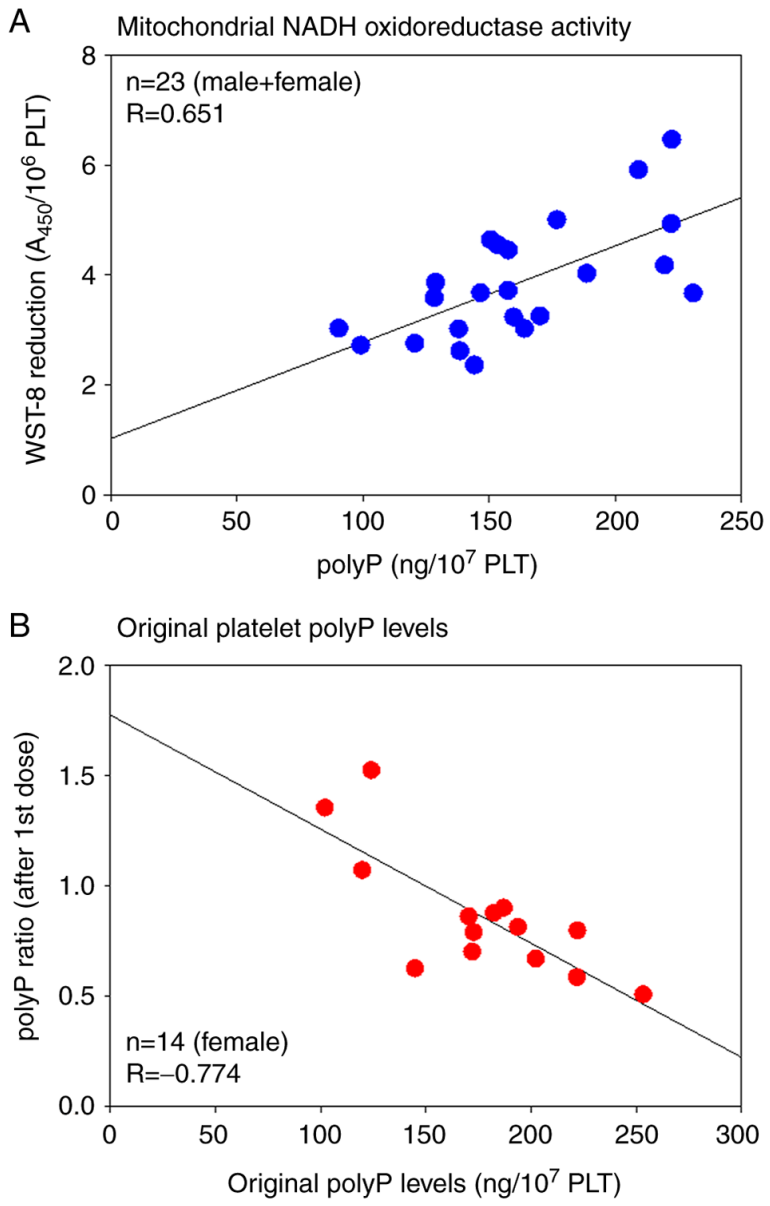

Figure 5. Scatter plots of the mitochondrial activity and polyP levels in the male + female group and the female group. (A) The relation between reduction in WST-8 activity and polyP levels, and (B) between polyP ratios and original polyP levels pre-first vaccination. The blue and red symbols represent 'males + females' and 'females alone', respectively. polyp, platelet polyphosphate.

( $\mathrm{R}=-0.774)$ was found between the polyP ratio and the original platelet polyP levels (Fig. 5B).

The effects of the Moderna vaccine (mRNA-1273) on polyP levels were examined in a small number of subjects only, due to its limited supply during our investigation (Table IV). A substantial reduction in platelet polyP levels was observed in three out of four female subjects after the first vaccination, whereas the polyP levels increased in three out of four subjects after the second vaccination. The oldest female subject was unique, and showed both a low side-effect score and COVID-19 arm soreness (37), both of which are usually observed after the first vaccination. A drastic increase in the side-effect score after the second vaccination was observed in only the youngest female subject.

\section{Discussion}

Prior to this study, it was hypothesized that if a micro-thrombosis forms in the peripheral tissue, most probably around the site of vaccine injection in the arm, then platelet polyP is released (and intra-platelet polyP levels thereafter decrease) to function as a coagulation factor. However, no symptoms indicating local thrombocytopenia or purpura were observed upon visual inspection. Although severe side-effects, classified as severe fever, headache and pain at the injection site, were reported in several female subjects after the second vaccination, it was speculated that the COVID-19 vaccination assessed likely does not induce platelet-dependent microthrombus formation or internal bleeding.

Interestingly, the Pfizer/BioNTech vaccine (BNT162b2) significantly reduced platelet polyP levels after the first vaccination, along with suppressed side-effects in the younger female group. Thus, a hypothesis to be considered for further investigation is that the spike protein produced by the mRNA vaccines induces inflammation, which is generally more severe in younger female individuals (38), and that the platelets activated by this inflammatory response release polyP, along with other anti-inflammatory molecules, to suppress the inflammation in a negative feedback loop. This hypothesis is illustrated in Fig. 6. At present, there is no evidence to suggest how these phenomena are related, necessitating further investigation to clarify the involvement of polyP in the inflammatory response initiated by the vaccine.

The dysfunction of this loop system after the second dose of the vaccine may be due to platelet status. Platelets, which contain various immunoreceptors, continuously scan for areas of injury or inflammation as versatile patrollers $(39,40)$. When platelets recognize intravascular pathogens, they directly limit pathogen growth and indirectly ensure pathogen clearance through the activation of immune cells $(3,6,40)$. Therefore, once platelet signals are received and taken over by immune cells, inflammation may not be regulated by platelets. However, if inflammation becomes more severe, platelets may become dysfunctional. This scenario could be explained by a 


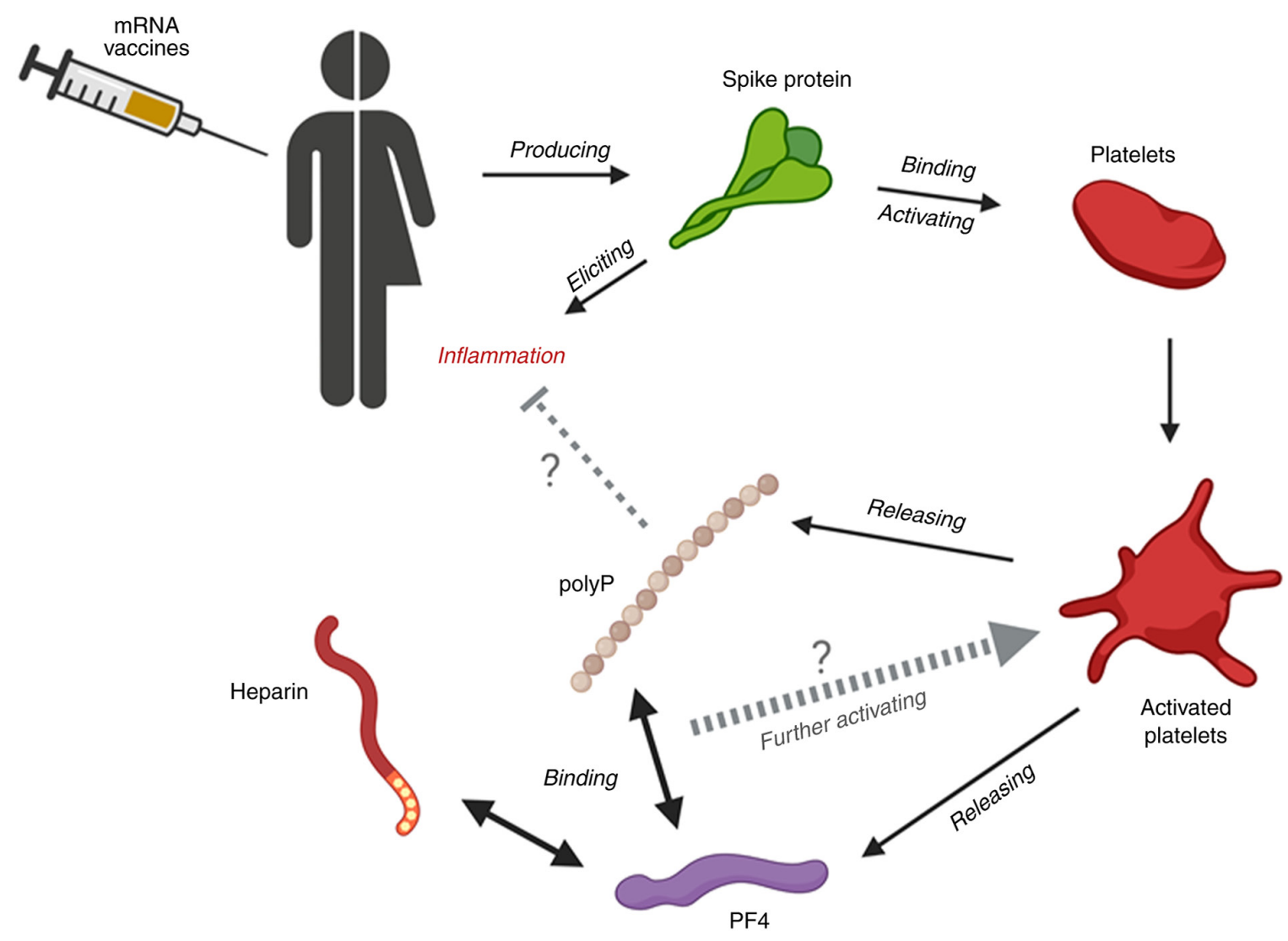

Figure 6. Schematic of the proposed functions of polyP released from activated platelets.

'chicken or egg' paradox-like essence, introduced by Portier and Campbell, in which aberrant platelet activation, which could be caused by continuous moderate (or severe) inflammation, can lead to predominantly inflammatory and thrombotic events (40).

It is important to evaluate the expression of platelet activation markers, such as CD62P, to confirm the release of polyP by activated platelets. However, to stabilize polyP levels, it is necessary to incubate blood samples at least overnight at $22-25^{\circ} \mathrm{C}$ (30). Furthermore, it was difficult to normalize the venipuncture skill, which can easily influence platelet conditions; thus, platelet activation was not evaluated in the present study.

The aforementioned explanation assumes that the reduction of platelet polyP is due to the release of polyP from activated platelets. Before discussing other possible explanations, polyP metabolism in platelets should be confirmed. It is generally accepted that polyP is synthesized from ATP or GTP and degraded by cellular enzymes in bacteria $(26,41,42)$. In eukaryotes, this mechanism is not yet fully understood; however, polyP is suggested to be synthesized and degraded by the same or a similar mechanism, given that inorganic phosphate $(\mathrm{Pi})$ is incorporated by a specific transporter into mammalian cells and used for polyP synthesis in mitochondria non-enzymatically (24). Although polyP is widely distributed in the cytoplasm and among organelles, it is most condensed when stored in dense granules in platelets and released upon activation (24). Thus, it is generally accepted that platelet polyP levels are regulated by three major factors: Activation levels, mitochondrial activity and extracellular Pi levels.
Since platelets of patients with sepsis or cardiac shock possess lower mitochondrial NADH levels (43), in this study, the activity of NADH using was evaluated using WST-8, a tetrazolium salt that is reduced to soluble formazan (44), and found a moderate correlation between the activity of this enzyme and polyP levels. Serum phosphate levels were not examined in this study, as no declarations for hyperphosphatemia or hypophosphatemia were obtained from individual participants. Taken together, these results suggest that basal polyP levels depend on mitochondrial activity, and influence the rate of reduction of polyP induced by platelet activation. Considering that platelet activation is a highly energy-dependent process (45), it is plausible that platelets with higher mitochondrial activity show stronger immune responses to released polyP, resulting in polyP reduction.

In clinical observations, SARS-CoV-2 infection initially produces inflammation, platelet activation, and prominent elevation of fibrinogen and D-dimer (10-12). Subsequently, the virus leads to various levels of thrombotic events and thrombocytopenia (10). The mRNA vaccines were designed to temporarily produce the spike protein at lower levels than those observed in SARS-CoV-2 infection or DNA vaccines. Thus, even though the initial events induced by SARS-CoV-2 can be reproduced by mRNA vaccines, severe symptoms observed in COVID-19 patients cannot be produced. However, due to individual differences in immune activity, vaccines sometimes induce thrombotic thrombocytopenia (VITT) $(46,47)$. To date, the incidence of VITT has been much lower in the case of mRNA vaccines than in DNA vaccines, such as ChAdOx1 nCov-19 and Ad26.COV2.5, wherein VITT has been reported in various groups and countries (29). 
Recently, the primary mechanism of VITT has been explained by heparin-induced thrombocytopenia (48). Platelet factor 4 (PF4) released from activated platelets binds blood heparin to form the complex (PF4-heparin), which binds to IgG antibodies, then subsequently binds to platelet Fc $\gamma$ RIIa receptors to further activate platelets to induce thrombosis (49-52). Interestingly, the authors who reported on this mechanism also mentioned the possibility that polyanions, such as polyP, may bind to PF4 to form the PF4-polyP complex and induce thrombosis (50). This possibility and the subsequent biological reactions should be further studied.

As described above, the possible anti-inflammatory effects of polyP, which are thought to be mediated through the suppression of the complement system, are discussed $(53,54)$. However, the conflicting possibility that polyP has the potential to induce thrombosis should be noted. Surprisingly, although the chain length of polyP may be a matter for further investigation, it has been reported that polyanions such as polyP can form a complex with PF4, and that this complex activates platelets, similar to the PF4-heparin complex $(48,50)$. Platelets also release anti-inflammatory cytokines, such as IL-4 and IL-10, upon activation (1); thus, PRP has been clinically utilized as an anti-inflammatory or a pain-relieving agent (55). At present, it is difficult to predict the risk of PF4-polyP complex-induced thrombosis by the autocrine loop or by exposure to bacterial polyP after vaccination.

By applying this possibility to the findings of this study, the biological significance of these conflicting functions could be interpreted as the unexpected aberrant activation and destruction of platelets, as well as the uncontrolled exacerbation of inflammation; thus, the immune system may be protected by the dual functions of platelets and polyP. However, especially in younger female subjects who generally exhibit stronger immune responses, exposure to pathogens or sustained inflammatory conditions for a long time (i.e., the interval between the first and second vaccination in this study $\sim 3$ weeks) may cause platelets to lose their ability to detect pathogens and allow the exacerbation of inflammation, which is recognized as a side-effect.

There are several limitations in the present study. First, most allocation guidelines have prioritized high-risk healthcare workers in most countries (56), including Japan. However, the vaccination schedule has been strictly controlled by the central and local governments, limiting the availability of a more diverse cohort. Second, our limited capacity to attain samples made it difficult to secure a sufficient number of subjects. As a result, it was not possible to expand the sample size to what was originally anticipated. Third, to support the specificity of the quantification data, it would be better to further examine the localization and the appearance of DAPI-dependent signals using a histochemical method that we previously performed for $\mathrm{Ca}^{2+}$-activated platelets $(30,32)$. Therefore, further investigation with a larger sample size is required, along with visualization and image analysis, to reach a definitive conclusion. However, the present findings regarding sex differences are essentially consistent with the previous reports of antibody formation (57-59), and therefore, this pilot study should be considered important for the scientific community.

In conclusion, these findings suggest that polyP released from activated platelets may be involved in the suppression of severe side-effects after the first vaccination in young female subjects. This may be related to the result that young female subjects complained of severe side-effects after the second vaccination, but not after the first vaccination. To control such high immune responses, more potent 'braking' systems are required to be balanced with potent 'accelerating' systems; PolyP may be a possible candidate factor.

\section{Acknowledgements}

Not applicable.

\section{Funding}

This work was supported by Japan Society for the Promotion of Science (Tokyo, Japan) Grants-in-Aid for Scientific Research [JSPS KAKENHI (grant no. 21K09932)].

\section{Availability of data and materials}

The datasets used and/or analyzed during the present study are available from the corresponding author on reasonable request.

\section{Authors' contributions}

TU and TK conceived the study. TK designed the study. TTs, TTa and KI assisted in the design of the study. TW analyzed the data. TU, AS, HA, TTs, TW, KI, HK, YK and TK performed the experiments. HA and YK collected the data. TU, HA, TTs, TTa and TK wrote the manuscript. TU, TTs, TTa and TK edited the manuscript. All authors have read and approved the final manuscript.

\section{Ethics approval and consent to participate}

The study design and consent forms for all procedures (approval no. 2019-0423) were approved by the Ethics Committee for Human Participants at the Niigata University School of Medicine (Niigata, Japan) and complied with the Helsinki Declaration of 1964, as revised in 2013.

\section{Patient consent for publication}

Written informed consent was obtained from all the patients for publication of their data.

\section{Competing interests}

The authors declare that they have no competing interests.

\section{References}

1. Chen Y, Zhong H, Zhao Y, Luo X and Gao W: Role of platelet biomarkers in inflammatory response. Biomark Res 8: 28, 2020.

2. Li C, Li J and Ni H: Crosstalk between platelets and microbial pathogens. Front Immunol 11: 1962, 2020.

3. Margraf A and Zarbock A: Platelets in Inflammation and Resolution. J Immunol 203: 2357-2367, 2019.

4. Morrell CN, Aggrey AA, Chapman LM and Modjeski KL: Emerging roles for platelets as immune and inflammatory cells. Blood 123: 2759-2767, 2014.

5. Gómez RM, López Ortiz AO and Schattner M: New roles of platelets in inflammation. Curr Opin Physiol 19: 99-104, 2021. 
6. Gros A, Ollivier V and Ho-Tin-Noé B: Platelets in inflammation: Regulation of leukocyte activities and vascular repair. Front Immunol 5: 678, 2014.

7. Wang MY, Zhao R, Gao LJ, Gao XF, Wang DP and Cao JM: SARS-CoV-2: Structure, biology, and structure-based therapeutics development. Front Cell Infect Microbiol 10: 587269, 2020.

8. Cox D: Targeting SARS-CoV-2-platelet interactions in COVID-19 and vaccine-related thrombosis. Front Pharmacol 12: 708665, 2021.

9. Lichtenberger LM and Vijayan KV: Is COVID-19-induced platelet activation a cause of concern for patients with cancer? Cancer Res 81: 1209-1211, 2021.

10. Wool GD and Miller JL: The impact of COVID-19 disease on platelets and coagulation. Pathobiology 88: 15-27, 2021.

11. Comer SP, Cullivan S, Szklanna PB, Weiss L, Cullen S Kelliher S, Smolenski A, Murphy C, Altaie H, Curran J, et al: COVID-19 induces a hyperactive phenotype in circulating platelets. PLoS Biol 19: e3001109, 2021.

12. Hottz ED, Azevedo-Quintanilha IG, Palhinha L, Teixeira L, Barreto EA, Pão CRR, Righy C, Franco S, Souza TML, Kurtz P, et al: Platelet activation and platelet-monocyte aggregate formation trigger tissue factor expression in patients with severe COVID-19. Blood 136: 1330-1341, 2020.

13. Yatim N, Boussier J, Chocron R, Hadjadj J, Philippe A Gendron N, Barnabei L, Charbit B, Szwebel TA, Carlier N, et al: Platelet activation in critically ill COVID-19 patients. Ann Intensive Care 11: 113, 2021

14. World Health Organization (WHO): WHO issues its first emergency use validation for a COVID-19 vaccine and emphasizes need for equitable global access. WHO, Geneva, 2020 https://www.who.int/news/item/31-12-2020-who-issues-its-first -emergency-use-validation-for-a-covid-19-vaccine-and-emphasizes-need-for-equitable-global-access. Accessed December 18 2021.

15. U.S. Food \& Drug Administration (FDA): FDA Approves First COVID-19 Vaccine. FDA, Silver Spring, MD, 2021. https://www.fda.gov/news-events/press-announcements/fda-approves-first-covid-19-vaccine. Accessed December 18, 2021.

16. Pharmaceutical Technology: COVID-19 vaccine market set to reach $\$ 19.5$ bn by 2026-register for free webinar. Pharmaceutical Technology, New York, NY, 2021. https://www.pharmaceutical-technology.com/news/covid-19-vaccine-market-set-to-reach19-5bn-by-2026-register-for-free-webinar/. Accessed December 18, 2021.

17. Nikkei Asia: Status of vaccinations around the world. Nikkei Inc., Tokyo, 2021. https://vdata.nikkei.com/en/newsgraphics/coronavirus-vaccine-status/. Accessed December 18, 2021.

18. Corbett KS, Edwards DK, Leist SR, Abiona OM, Boyoglu-Barnum S, Gillespie RA, Himansu S, Schäfer A, Ziwawo CT, DiPiazza AT, et al: SARS-CoV-2 mRNA vaccine design enabled by prototype pathogen preparedness. Nature 586 567-571, 2020

19. Jalkanen $P$, Kolehmainen $P$, Häkkinen HK, Huttunen $M$ Tähtinen PA, Lundberg R, Maljanen S, Reinholm A, Tauriainen S, Pakkanen SH, et al: COVID-19 mRNA vaccine induced antibody responses against three SARS-CoV-2 variants. Nat Commun 12 : $3991,2021$.

20. Menni C, Klaser K, May A, Polidori L, Capdevila J, Louca P, Sudre CH, Nguyen LH, Drew DA, Merino J, et al: Vaccine side-effects and SARS-CoV-2 infection after vaccination in users of the COVID Symptom Study app in the UK: A prospective observational study. Lancet Infect Dis 21: 939-949, 2021.

21. Morrissey JH, Choi SH and Smith SA: Polyphosphate: An ancient molecule that links platelets, coagulation, and inflammation. Blood 119: 5972-5979, 2012.

22. Travers RJ, Smith SA and Morrissey JH: Polyphosphate, platelets, and coagulation. Int J Lab Hematol 37 (Suppl 1): S31-S35, 2015.

23. Weitz JI and Fredenburgh JC: Platelet polyphosphate: The long and the short of it. Blood 129: 1574-1575, 2017.

24. Abbasian N and Harper MT: High extracellular phosphate increases platelet polyphosphate content. Platelets 32: 992-994, 2021.

25. Baker CJ, Smith SA and Morrissey JH: Polyphosphate in thrombosis, hemostasis, and inflammation. Res Pract Thromb Haemost 3: 18-25, 2019.

26. Morrissey JH: Polyphosphate: A link between platelets, coagulation and inflammation. Int J Hematol 95: 346-352, 2012.

27. World Medical Association: World Medical Association Declaration of Helsinki: Ethical principles for medical research involving human subjects. JAMA 310: 2191-2194, 2013
28. Kurihara C: The 2013 revision of the declaration of Helsinki: Challenges for the Next 50 Years. Rinsho Yakuri/Jap J Clin Pharmacol Ther 45: 41-51, 2014.

29. The Japan Times: Japan's COVID-19 vaccine plan prioritizes health care workers and older residents. The Japan Times, Ltd., Tokyo, 2020. https://www.japantimes. co.jp/news/2020/ $12 / 25 /$ national/japan-vaccine-older-people/. Accessed December 18, 2021. (This page is now missing).

30. Watanabe T, Kitamura Y, Aizawa H, Masuki H, Tsujino T, Sato A, Kawabata H, Isobe K, Nakata K and Kawase T: Fluorometric Quantification of human platelet polyphosphate using 4',6-diamidine-2-phenylindole Dihydrochloride: Applications in the Japanese population. Int J Mol Sci 22: 7257, 2021.

31. Vollmar B, Slotta JE, Nickels RM, Wenzel E and Menger MD: Comparative analysis of platelet isolation techniques for the in vivo study of the microcirculation. Microcirculation 10 : 143-152, 2003.

32. Sato A, Aizawa H, Tsujino T, Isobe $\mathrm{K}$, Watanabe T, Kitamura $\mathrm{Y}$ and Kawase T: Fluorescent Cytochemical detection of polyphosphates associated with human platelets. Int J Mol Sci 22: 1040,2021.

33. Aschar-Sobbi R, Abramov AY, Diao C, Kargacin ME, Kargacin GJ, French RJ and Pavlov E: High sensitivity, quantitative measurements of polyphosphate using a new DAPI-based approach. J Fluoresc 18: 859-866, 2008.

34. Baev AY, Angelova PR and Abramov AY: Inorganic polyphosphate is produced and hydrolyzed in F0F1-ATP synthase of mammalian mitochondria. Biochemical J 477: 1515-1524, 2020.

35. Lynn WS and Brown RH: Synthesis of polyphosphate by rat liver mitochondria. Biochem Biophys Res Commun 11: 367-371, 1963

36. Müller WEG, Schröder HC and Wang X: Inorganic polyphosphates as storage for and generator of metabolic energy in the extracellular matrix. Chem Rev 119: 12337-12374, 2019.

37. Blumenthal KG, Freeman EE, Saff RR, Robinson LB, Wolfson AR, Foreman RK, Hashimoto D, Banerji A, Li L, Anvari S and Shenoy ES: Delayed large local reactions to mRNA-1273 Vaccine against SARS-CoV-2. N Engl J Med 384: 1273-1277, 2021

38. Chen PW, Tsai ZY, Chao TH, Li YH, Hou CJ and Liu PY: Addressing vaccine-induced immune thrombotic thrombocytopenia (VITT) Following COVID-19 vaccination: A Mini-review of practical strategies. Acta Cardiol Sin 37: 355-364, 2021

39. Guo L and Rondina MT: The era of thromboinflammation: Platelets are dynamic sensors and effector cells during infectious diseases. Front Immunol 10: 2204, 2019.

40. Portier I and Campbell RA: Role of platelets in detection and regulation of infection. Arterioscler Thromb Vasc Biol 41: 70-78, 2021.

41. Simbulan-Rosenthal CM, Carney BC, Gaur A, Moghe M, Crooke E, Moffatt LT, Shupp JW and Rosenthal DS: Inorganic polyphosphates are important for cell survival and motility of human skin keratinocytes and play a role in wound healing. In: Contemporary Topics about Phosphorus in Biology and Materials. Churchill DG, Sikirić MD, Čolović B and Milhofer HF (eds). IntechOpen, London, 2019.

42. Simbulan-Rosenthal CM, Gaur A, Sanabria VA, Dussan LJ, Saxena R, Schmidt J, Kitani T, Chen YS, Rahim S, Uren A, et al: Inorganic polyphosphates are important for cell survival and motility of human skin keratinocytes. Exp Dermatol 24: 636-639, 2015.

43. Protti A, Fortunato F, Artoni A, Lecchi A, Motta G, Mistraletti G, Novembrino C, Comi GP and Gattinoni L: Platelet mitochondrial dysfunction in critically ill patients: Comparison between sepsis and cardiogenic shock. Crit Care 19: 39, 2015.

44. Ishiyama M, Miyazono Y, Sasamoto K, Ohkura Y and Ueno K: A highly water-soluble disulfonated tetrazolium salt as a chromogenic indicator for NADH as well as cell viability. Talanta 44: $1299-1305,1997$.

45. Aibibula M, Naseem KM and Sturmey RG: Glucose metabolism and metabolic flexibility in blood platelets. J Thromb Haemost 16: 2300-2314, 2018.

46. Arepally GM and Ortel TL: Vaccine-induced immune thrombotic thrombocytopenia: What we know and do not know. Blood 138: 293-298, 2021

47. Huynh A, Kelton JG, Arnold DM, Daka M and Nazy I: Antibody epitopes in vaccine-induced immune thrombotic thrombocytopaenia. Nature 596: 565-569, 2021.

48. Novak N, Tordesillas L and Cabanillas B: Adverse rare events to vaccines for COVID-19: From hypersensitivity reactions to thrombosis and thrombocytopenia. Int Rev Immunol: Jul 12, 2021 (Epub ahead-of print). 
49. Arepally GM and Padmanabhan A: Heparin-induced thrombocytopenia. Arterioscler Thromb Vasc Biol 41: 141-152, 2021.

50. Cines DB, Yarovoi SV, Zaitsev SV, Lebedeva T, Rauova L, Poncz M, Arepally GM, Khandelwal S, Stepanova V, Rux AH, et al: Polyphosphate/platelet factor 4 complexes can mediate heparin-independent platelet activation in heparin-induced thrombocytopenia. Blood Adv 1: 62-74, 2016.

51. Prechel MM and Walenga JM: Emphasis on the Role of PF4 in the incidence, pathophysiology and treatment of heparin induced thrombocytopenia. Thromb J 11: 7, 2013.

52. Rauova L, Zhai L, Kowalska MA, Arepally GM, Cines DB and Poncz M: Role of platelet surface PF4 antigenic complexes in heparin-induced thrombocytopenia pathogenesis: Diagnostic and therapeutic implications. Blood 107: 2346-2353, 2006.

53. Conway EM: Polyphosphates and complement activation. Front Med (Lausanne) 6: 67, 2019.

54. Wat JM, Foley JH, Krisinger MJ, Ocariza LM, Lei V, Wasney GA, Lameignere E, Strynadka NC, Smith SA, Morrissey JH and Conway EM: Polyphosphate suppresses complement via the terminal pathway. Blood 123: 768-776, 2014.
55. Kuffler DP: Variables affecting the potential efficacy of PRP in providing chronic pain relief. J Pain Res 12: 109-116, 2019.

56. Ubel PA: Vaccinating health care employees-do they all deserve early access? N Engl J Med 384: e39, 2021.

57. Ciarambino $\mathrm{T}$, Para $\mathrm{O}$ and Giordano $\mathrm{M}$ : Immune system and COVID-19 by sex differences and age. Womens Health (Lond) 17: 17455065211022262, 2021.

58. Lo Sasso B, Giglio RV, Vidali M, Scazzone C, Bivona G, Gambino CM, Ciaccio AM, Agnello L and Ciaccio M: Evaluation of Anti-SARS-Cov-2 S-RBD IgG antibodies after COVID-19 mRNA BNT162b2 vaccine. Diagnostics (Basel) 11: 1135, 2021.

59. Pellini R, Venuti A, Pimpinelli F, Abril E, Blandino G, Campo F Conti L, De Virgilio A, De Marco F, Di Domenico EG, et al: Initial observations on age, gender, BMI and hypertension in antibody responses to SARS-CoV-2 BNT162b2 vaccine. EClinicalMedicine 36: 100928, 2021.

This work is licensed under a Creative Commons Attribution-NonCommercial-NoDerivatives 4.0 International (CC BY-NC-ND 4.0) License. 